\title{
Versatile, highly reconfigurable, high bandwidth, radio frequency and microwave photonic Hilbert transformers with soliton crystal Kerr micro-comb sources
}

david moss ( $\square$ dmoss@swin.edu.au )

Swinburne University of Technology https://orcid.org/0000-0001-5195-1744

\section{Research Article}

Keywords: Kerr frequency comb, Hilbert transform, integrated optics, all-optical signal processing

Posted Date: February 9th, 2022

DOI: https://doi.org/10.21203/rs.3.rs-1340864/v1

License: (9) This work is licensed under a Creative Commons Attribution 4.0 International License.

Read Full License 


\title{
Versatile, highly reconfigurable, high bandwidth, radio frequency and microwave photonic Hilbert transformers with soliton crystal Kerr micro-comb sources
}

\author{
David J. Moss \\ ${ }^{a}$ Optical Sciences Centre, Swinburne University of Technology, Hawthorn, VIC 3122, Australia
}

\begin{abstract}
We experimentally demonstrate bandwidth-tunable RF photonic Hilbert transformer based on an integrated Kerr microcomb source. The micro-comb is generated by an integrated micro-ring resonator with a free spectral range of $48.9 \mathrm{GHz}$, yielding 75 micro-comb lines in the telecom C-band. By programming and shaping the generated comb lines according to calculated tap weights, we demonstrate high-speed Hilbert transform functions with tunable bandwidths ranging from 1.2 $\mathrm{GHz}$ to $15.3 \mathrm{GHz}$, switchable center frequencies from baseband to $9.5 \mathrm{GHz}$, and arbitrary fractional orders. The experimental results show good agreement with theory and confirm the effectiveness of our approach.
\end{abstract}

Keywords: Kerr frequency comb, Hilbert transform, integrated optics, all-optical signal processing.

\section{INTRODUCTION}

The Hilbert transform is a fundamental signal processing function with wide range of applications in radar systems, signal sideband modulators, measurement systems, signal sampling, and many others [1-8]. Fractional Hilbert transforms provide an additional degree of freedom in terms of a variable phase shift, which can meet the special requirements of unilateral communication [2] and confidentiality of hardware keys [3]. In practical applications such as multiplexing and demultiplexing signals, analyzing individual sub-channel spectral components, etc., Hilbert transformers are typically realized as a truncated or windowed version of the ideal Hilbert transform impulse response [5-7]. Therefore, Hilbert transformers covering a wide range of different band pass regions are highly desirable.

Compared to electrical Hilbert transformers that suffer from intrinsic bandwidth bottlenecks, photonic integrated devices have shown advantages in high-speed signal processing. RF photonic Hilbert transformers have been proposed based on fiber Bragg gratings [9-15], micro-ring / micro-disk resonators [16, 17], and integrated reconfigurable microwave processors [18]. However, most of these schemes focus on generating the Hilbert transform of the complex optical fields rather than the actual RF signal. In order to realize highly reconfigurable RF photonic Hilbert transformers, transversal schemes with a high reconfigurability have been investigated $[19,20]$. However, the use of multiple discrete laser sources presents limitations in the overall system footprint, processing performance, and the potential for full monolithic integration.

Recently, micro-combs that offer a large number of coherent wavelengths from one compact device have attracted significant interest as a fundamentally powerful tool for microwave processing $[8,21-40]$. They have been used for various advanced signal processing [8, 41-64] and neural networks [65-67]. In Ref. [1], we reported a fractional Hilbert transformer with a RF bandwidth ranging from 5 to 9 octaves depending on the fractional order. It was based on a soliton crystal microcomb with $\mathrm{FSR}=48.9 \mathrm{GHz}$, and 17 taps were selected from the 75 wavelengths generated in the $\mathrm{C}$ band as discrete taps. 
In this paper, we further demonstrate a photonic Hilbert transformer with variable bandwidth and RF center frequency [1]. It is based on a transversal filter system with a soliton crystal Kerr micro-comb source. In the experimental demonstration, we used $40 \mathrm{comb}$ lines in the C-band [1]. By programming and shaping the comb lines according to calculated tap weights, the center frequency of Hilbert transform was tuned from baseband to $9.5 \mathrm{GHz}$, and the bandwidth of RF amplitude and phase responses was tuned from 1.2 to $15.3 \mathrm{GHz}$, confirming the high reconfigurability of our system. The experimental results show good agreement with the theory, confirming the feasibility of our approach towards the realization of highspeed reconfigurable Hilbert transformers with reduced footprint, lower complexity, and potentially reduced cost.

\section{OPERATION PRINCIPLE}

The figure. 1 shows is a schematic diagram of a Hilbert transformer based on a micro-comb. The micro-comb is produced on a high-Q MRR by pumping. The CW laser is used and the EDFA is used to amplify the MRR whose polarization state is aligned with the TE mode. When the pump wavelength is manually swept across one of the resonances of the MRR and the pump power is large enough to generate sufficient parameter gain, the optical parameter oscillation will occur, and finally a Kerr frequency comb with a spacing equal to the MRR free spectral range will be generated.

The spectral transfer function of a general fractional Hilbert transformer is given by $[1,8]$ :

$$
H_{P}(\omega)=\left\{\begin{array}{c}
e^{-j \varphi}, \text { if } 0 \leq \omega<\pi \\
e^{j \varphi}, \text { if }-\pi \leq \omega<0
\end{array}\right.
$$

where $j=\sqrt{-1}, \varphi=P \times \pi / 2$ denotes the phase shift, $P$ is the fractional order (when $P=1$, it becomes a standard Hilbert transformer). The corresponding impulse response is given by a continuous hyperbolic function:

$$
h_{P}(t)=\left\{\begin{array}{c}
\frac{1}{\pi t}, t \neq 0 \\
\cot (\varphi), t=0
\end{array}\right.
$$

This hyperbolic function is truncated and sampled in time with discrete taps for digital implementation. The null frequency is given by:

$$
f_{c}=1 / \Delta t
$$

where $\Delta \mathrm{t}$ denotes the sample spacing. The coefficient of the tap at $\mathrm{t}=0$ can be adjusted to achieve a tunable fractional order [1]. The normalized power of each comb line is:

$$
P_{n}=\frac{1}{\pi\left|n-\frac{N}{2}+0.5\right|}
$$

where $\mathrm{N}$ is the number of comb lines, or taps, and $\mathrm{n}=0,1,2, \ldots, N-1$ is the comb index. 


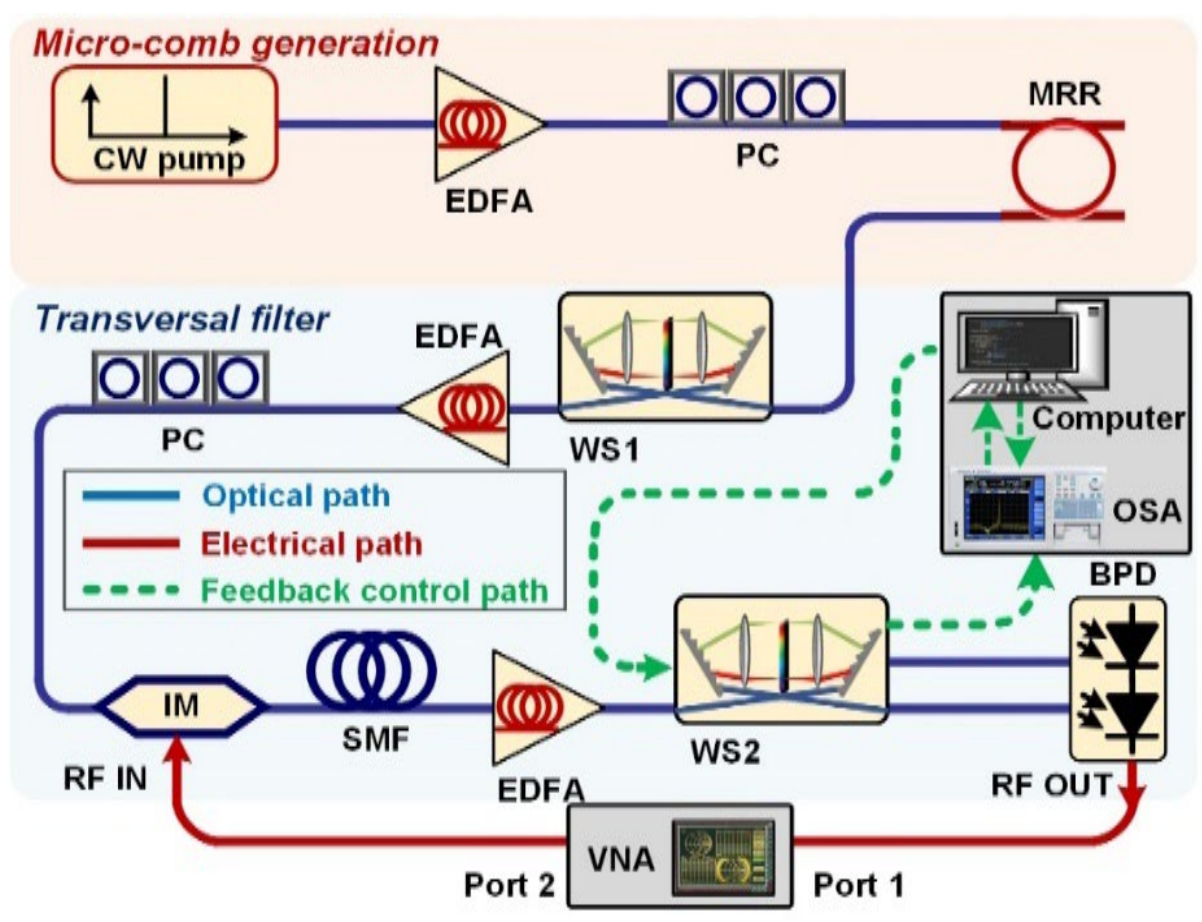

Fig. 1. Schematic diagram of fractional Hilbert transformer based on an integrated Kerr micro-comb source. EDFA: erbium-doped fiber amplifier. PC: polarization controller. MRR: micro-ring resonator. WS: WaveShaper. IM: Intensity modulator. SMF: single mode fiber. OSA: optical spectrum analyzer. BPD: Balanced photodetector. VNA: vector network analyzer.

In order to scale the bandwidth of the standard and fractional Hilbert transformers, we design the spectral transfer function of the Hilbert transformer through the Remez algorithm [69], and change the operating bandwidth by multiplying the corresponding impulse response with the cosine function. Therefore, the resulting discrete impulse response becomes:

$$
h_{T B W H T}(n)=P_{n} \cdot \cos \left(2 \pi n \cdot f_{B W}\right)
$$

where $\mathrm{fBW}$ is the scalable bandwidth. To further switch the centre frequency of the Hilbert transformer, the tap coefficients were multiplied by a sine function to shift the RF transmission spectrum. The corresponding discrete impulse response is given by

$$
h_{T C F H T}(n)=P_{n} \cdot \sin \left(2 \pi n \cdot f_{B W}\right)
$$

we use the transverse method to realize the Hilbert transformer with RF center frequency and variable bandwidth. The transfer function $[1,8,9,70-78]$ can be described as:

$$
F(\omega)=\sum_{n-0}^{M-1} h(n) e^{-j \omega n T}
$$

where $\mathrm{M}$ is the number of taps, $\omega$ is the RF angular frequency, $\mathrm{T}$ is the time delay between adjacent taps, and $\mathrm{h}(\mathrm{n})$ is the tap coefficient of the nth tap. 


\section{EXPERIMENTAL RESULTS}

The MRR used here is manufactured on the platform based on Hydex glass [30, 33, 34, 38, 39] using CMOS manufacturing process. First, use PECVD to deposit Hydex film ( $\mathrm{n}=\sim 1.7$ at $1550 \mathrm{~nm}$ ), and then pattern by deep ultraviolet (UV) and reactive ion etching [79] to obtain waveguides with very low surface roughness. Finally, the upper cladding layer composed of silicon dioxide $(\mathrm{n}=\sim 1.44$ at $1550 \mathrm{~nm})$ is deposited. The main advantage of our platform is that it has ultra-low linear loss $(\sim 0.06 \mathrm{~dB} \cdot \mathrm{cm}-1)$ and moderately high optical nonlinear parameters $(\sim 233 \mathrm{~W}-1 \cdot \mathrm{km}-1)$. Because the platform has ultra-low loss, our MRR has a $\mathrm{Q}$ factor of up to about 1.5 million. The radius of the MRR is $\sim 592 \mu \mathrm{m}$, which corresponds to an optical FSR of $0.393 \mathrm{~nm}$ or $48.9 \mathrm{GHz}$. Such a small FSR greatly increases the number of wavelengths available on the C-band, up to 75 wavelengths, which is more than twice that of our previous results [58].

In order to generate the micro-comb, the $\mathrm{CW}$ pump power is amplified to $\sim 30.5 \mathrm{dBm}$. When the detuning between the pump wavelength and the cold resonance of the MRR becomes small enough so that the cavity power reaches the threshold, modulation instability will occur (MI) driven oscillation [23]. Then the main comb is generated, the spacing of which is determined by the MI gain peak value. As the detuning changes, it forms a spectrum similar to that reported by the spectral interference between the solitons that are closely packed in the cavity, that is, the soliton crystal [27-29]. This crystal is mainly used as the basis of radio frequency oscillators with low phase noise [80].

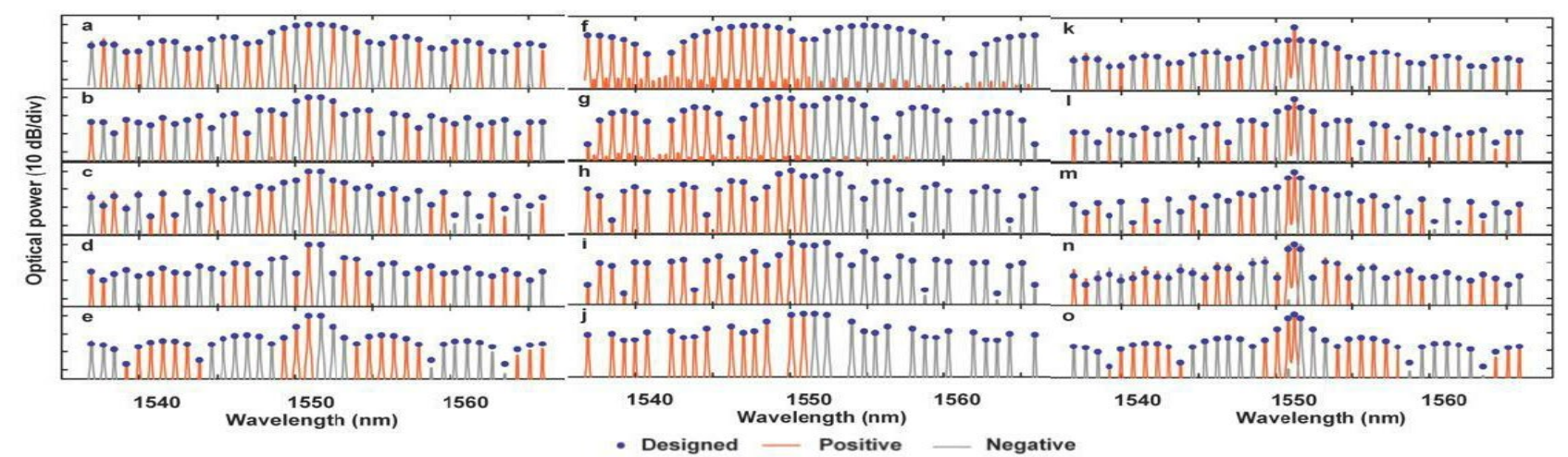

The soliton crystal is first flattened by a spectrum shaper (waveshaper 4000s), and then modulated with an RF input signal to broadcast the RF waveform to all wavelength channels at the same time, generating 75 replicas, but we only use 38 or 39 of them as taps. The $98 \mathrm{GHz}$ interval $(2 * 48.9 \mathrm{GHz})$, through 3.84 kilometers of standard single-film fiber (SMF), provides a progressive time delay between wavelengths. The fibre was approximately twice as long as that used in [58] in order to yield comparable RF bandwidths. The dispersion of the SMF was $\sim 17.4 \mathrm{ps} / \mathrm{nm} / \mathrm{km}$, corresponding to a time delay $\Delta t=26.25$ ps between adjacent wavelengths. Next, the second WaveShaper accurately shaped the comb power according to the designed tap coefficients, with the shaped comb spectrum shown in Fig. 2, for the integral order (90 degree phaseshift) tunable bandpass (a) (e), integral order ( 90 degree phaseshift) tunable lowpass (f) $\sim(j)$, and tunable fractional order ( 45 degree phaseshift) bandpass filter $(\mathrm{k}) \sim(\mathrm{o})$. Note that all devices used a $\sim 100 \mathrm{GHz}$ tap spacing (or more precisely 48.9 × $2=$ $97.8 \mathrm{GHz}$ ), yielding 39 tap lines across the C-band. Note the fractional order device used an extra single tap line at the centre wavelength, yielding 40 wavelengths overall, with the centre 3 wavelengths then being spaced at $48.9 \mathrm{GHz}$. The result was that the Nyquist zone for the fractional device was $24.5 \mathrm{GHz}$ rather than $48.9 \mathrm{GHz}$ for the integral order devices. For standard Hilbert transformer, this extra specific tap coefficient is not needed and so in principle the full $48.9 \mathrm{GHz}$ comb (75 lines over the C-band) could have been used although these results are not shown here. 


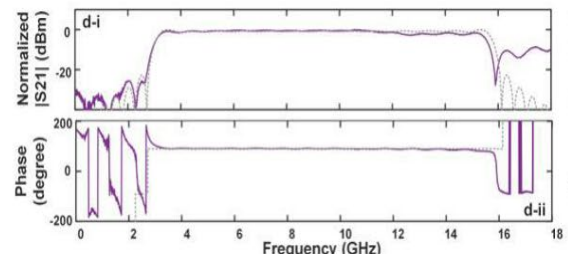

(a) $5.6 \mathrm{Ghz}$

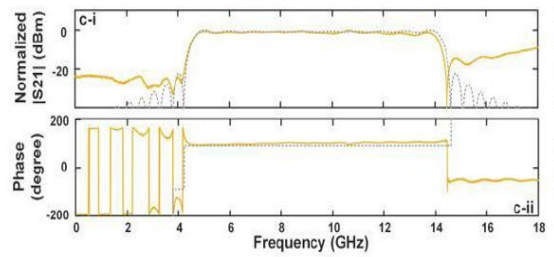

(d) $4.1 \mathrm{Ghz}$

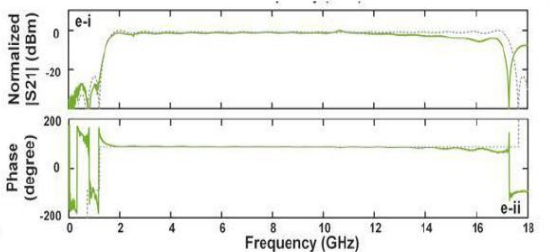

(b) $7.1 \mathrm{Ghz}$

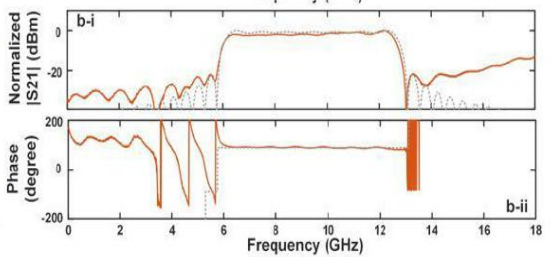

(e) $2.6 \mathrm{Ghz}$

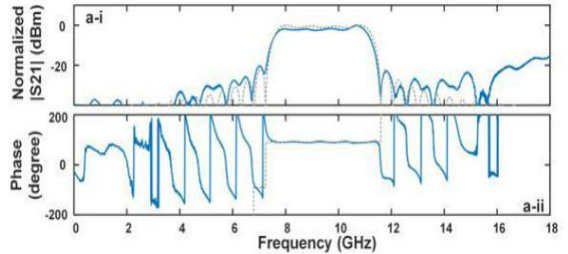

(c) $1.2 \mathrm{Ghz}$

The wavelength channels for both positive (solid red line) and negative (solid grey line) taps were separately measured by an optical spectrum analyser (OSA), achieving good performance that agreed well with the theory (blue dot). Finally, the weighted and delayed replicas were combined and converted back into the RF domain via a balanced photodetector (Finisar BPDV2150R).

The system RF frequency response was characterized with a calibrated vector network analyser (VNA, Agilent MS4644B) to measure the RF transmission and phase response. Fig. 3 (a-e) presents the simulated (dashed curves) and measured (solid curves) RF frequency response for both the magnitude and phase of the standard Hilbert transformer, yielding variable bandwidths ranging from 3.4 to $15.3 \mathrm{GHz}$. The centre frequency of the Hilbert transformer was set into half of the FSR $_{\text {RF, }}$ which was $19 / 2=9.5 \mathrm{GHz}$ in our case.

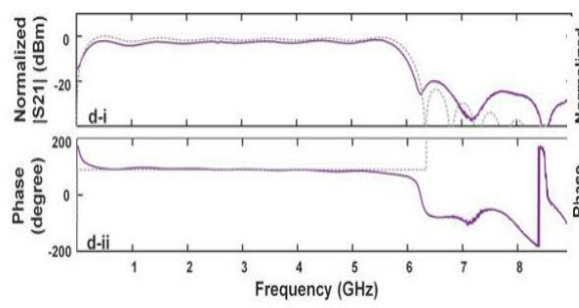

(a) $5.6 \mathrm{Ghz}$
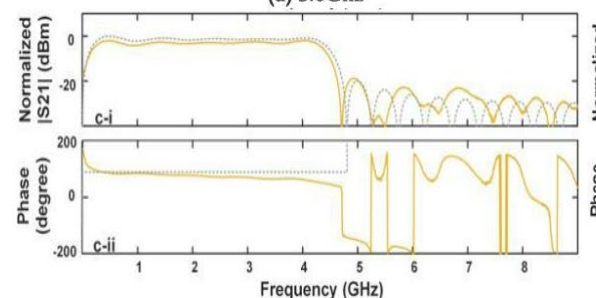

(d) $4.1 \mathrm{Ghz}$

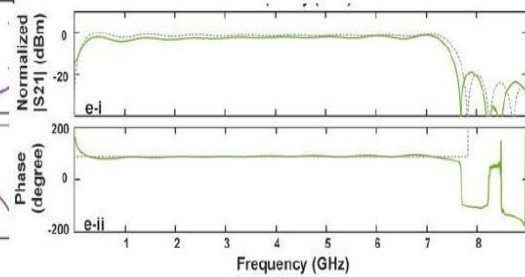

(b) $7.1 \mathrm{Ghz}$

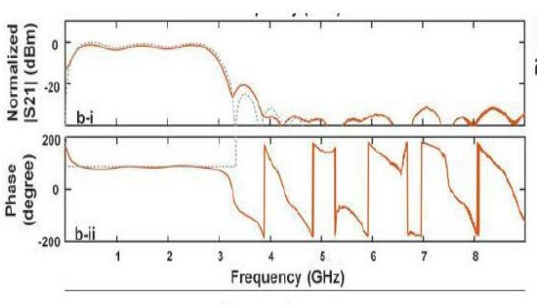

(e) $2.6 \mathrm{Ghz}$

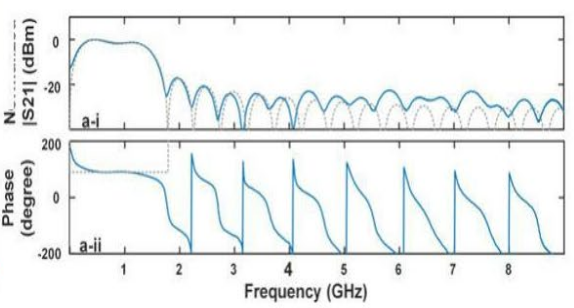

(c) $1.2 \mathrm{Ghz}$

Figure.4 shows the measured results for the RF amplitude and phase response of the lowpass Hilbert transformer, showing tunable bandwidths ranging from 1.2 to $7.1 \mathrm{GHz}$ that match closely with the simulated results. We also performed a demonstration of a fractional Hilbert transformer with switchable RF bandwidths ranging from 3.5 to $15.2 \mathrm{GHz}$. The 
simulated and measured RF amplitude and phase responses are shown in Fig. 6. We achieved a fractional order of 0.5, which corresponds to a 45-degree phase shift, using up to 39 wavelengths or taps. The increased number of taps used here compared with our previous work (39 here versus 17 taps in [58]) only resulted in an increase in overall bandwidth of about $0.6 \mathrm{GHz}$, although the performance in octaves was improved a bit more than this (5 octaves for 17 taps versus 6.3 octaves achieved here, as shown in Table I). These results have significant implications for the broader field of microwave photonics [81-127] including the wider use of micro-comb sources, [128-241] even potentially for applications in the midIR [242-248] since this approach has a wide range of microwave and RF applications and functions.

Table 1. Calculated performance of the standard Hilbert transformer

\begin{tabular}{|c|c|c|c|}
\hline & 4 taps & 16 taps & 38 taps \\
\hline Lower cutoff frequency $(\mathrm{GHz})$ & 16.3 & 17.9 & 18.2 \\
\hline Upper cutoff frequency $(\mathrm{GHz})$ & 2.2 & 0.5 & 0.2 \\
\hline 3-dB bandwidth $(\mathrm{GHz})$ & 14.1 & 17.4 & 18.4 \\
\hline Octaves & 2.9 & 5 & 0.1 \\
\hline
\end{tabular}

\section{CONCLUSION}

We demonstrate a photonic Hilbert transformer with variable bandwidth and RF center frequency. Up to 39 wavelengths or taps are used, enabling tunable bandwidths from 1.2 to $15.3 \mathrm{GHz}$ and switchable center frequencies from baseband to 9.5 GHz. Dynamic adjustment of bandwidth and center frequency is achieved by changing the tap weights. This microcomb-based approach provides a solid foundation for the realization of fully integrated photonic signal processors in future ultra-high-speed RF systems.

Competing interests: The authors declare no competing interests.

\section{REFERENCES}

[1] M. Tan, X. Xu, A. Boes, B. Corcoran, J. Wu, T. G. Nguyen, S. T. Chu, B. E. Little, A. J. Lowery, R. Morandotti, A. Mitchell, and D. J. Moss, "Highly Versatile Broadband RF Photonic Fractional Hilbert Transformer Based on a Kerr Soliton Crystal Microcomb," Journal of Lightwave Technology, vol. 39, no. 24, pp. 7581-7587, 2021.

[2] L. Moura, "Radio Frequency Implementation of the Fractional Hilbert Transform with Transversal Filters," Circuits, Systems \& Signal Processing, vol. 26, pp. 407-417, 2007.

[3] A. W. Lohmann, D. Mendlovic, and Z. Zalevsky, "Fractional Hilbert transform," Optics Letters, vol. 21, no. 4, pp. 281-283, 1996/02/15, 1996.

[4] A. D. Poularikas, A. F. Poularikas, and P. A. D, The Transforms and Applications Handbook: CRC-Press, 1996.

[5] V. K. Peddinti, and R. Kumaresan, "Bandpass phase shifter and analytic signal generator," Signal Process., vol. 125, no. C, pp. 216-220, 2016.

[6] H. P. Bazargani, M. d. R. Fernández-Ruiz, and J. Azaña, "Tunable, nondispersive optical filter using photonic Hilbert transformation," Optics Letters, vol. 39, no. 17, pp. 5232-5235, 2014/09/01, 2014.

[7] H. P. Bazargani, M. R. Fernández-Ruiz, and J. Azaña, "Tunable optical filter using photonic Hilbert transformation," OSA Technical Digest (online). p. SPM4D.6.

[8] T. G. Nguyen, M. Shoeiby, S. T. Chu, B. E. Little, R. Morandotti, A. Mitchell, and D. J. Moss, "Integrated frequency comb source based Hilbert transformer for wideband microwave photonic phase analysis," Optics Express, vol. 23, no. 17, pp. 22087-22097, 2015/08/24, 2015.

[9] H. Emami, N. Sarkhosh, L. A. Bui, and A. Mitchell, "Wideband RF photonic in-phase and quadrature-phase generation," Optics Letters, vol. 33, no. 2, pp. 98-100, 2008/01/15, 2008.

[10] M. Li, and J. Yao, "All-fiber temporal photonic fractional Hilbert transformer based on a directly designed fiber Bragg grating,” Optics Letters, vol. 35, no. 2, pp. 223-225, 2010/01/15, 2010. 
[11] M. Li, and J. Yao, "Experimental Demonstration of a Wideband Photonic Temporal Hilbert Transformer Based on a Single Fiber Bragg Grating,” IEEE Photonics Technology Letters, vol. 22, no. 21, pp. 1559-1561, 2010.

[12] M. H. Asghari, and J. Azaña, "All-optical Hilbert transformer based on a single phase-shifted fiber Bragg grating: design and analysis," Optics Letters, vol. 34, no. 3, pp. 334-336, 2009/02/01, 2009.

[13] T. Yang, J. Dong, L. Liu, S. Liao, S. Tan, L. Shi, D. Gao, and X. Zhang, "Experimental observation of optical differentiation and optical Hilbert transformation using a single SOI microdisk chip," Scientific Reports, vol. 4, no. 1, pp. 3960, 2014/02/04, 2014.

[14] Z. Zhang, C. Sima, B. Liu, B. Cai, Y. Gao, M. Zhang, L. Shen, Y. Yu, M. Huang, Z. Lian, M. T. Posner, J. C. Gates, P. G. R. Smith, and D. Liu, "Wideband and continuously-tunable fractional photonic Hilbert transformer based on a single high-birefringence planar Bragg grating," Optics Express, vol. 26, no. 16, pp. 20450-20458, 2018/08/06, 2018.

[15] C. Sima, J. C. Gates, C. Holmes, P. L. Mennea, M. N. Zervas, and P. G. R. Smith, “Terahertz bandwidth photonic Hilbert transformers based on synthesized planar Bragg grating fabrication," Optics Letters, vol. 38, no. 17, pp. 3448-3451, 2013/09/01, 2013.

[16] L. Zhuang, M. R. Khan, W. Beeker, A. Leinse, R. Heideman, and C. Roeloffzen, "Novel microwave photonic fractional Hilbert transformer using a ring resonator-based optical all-pass filter," Optics Express, vol. 20, no. 24, pp. 26499-26510, 2012/11/19, 2012.

[17] H. Shahoei, P. Dumais, and J. Yao, "Continuously tunable photonic fractional Hilbert transformer using a highcontrast germanium-doped silica-on-silicon microring resonator," Optics Letters, vol. 39, no. 9, pp. 2778-2781, 2014/05/01, 2014.

[18] W. Liu, M. Li, R. S. Guzzon, E. J. Norberg, J. S. Parker, M. Lu, L. A. Coldren, and J. Yao, “A fully reconfigurable photonic integrated signal processor," Nature Photonics, vol. 10, no. 3, pp. 190-195, 2016/03/01, 2016.

[19] Z. Li, Y. Han, H. Chi, X. Zhang, and J. Yao, “A Continuously Tunable Microwave Fractional Hilbert Transformer Based on a Nonuniformly Spaced Photonic Microwave Delay-Line Filter,” Journal of Lightwave Technology, vol. 30, no. 12, pp. 1948-1953, 2012/06/15, 2012.

[20] Z. Li, H. Chi, X. Zhang, and J. Yao, “A Continuously Tunable Microwave Fractional Hilbert Transformer Based on a Photonic Microwave Delay-Line Filter Using a Polarization Modulator," IEEE Photonics Technology Letters, vol. 23, no. 22, pp. 1694-1696, 2011.

[21] M. Peccianti, M. Ferrera, L. Razzari, R. Morandotti, B. E. Little, S. T. Chu, and D. J. Moss, "Subpicosecond optical pulse compression via an integrated nonlinear chirper," Optics Express, vol. 18, no. 8, pp. 7625-7633, 2010/04/12, 2010.

[22] D. Duchesne, M. Peccianti, M. R. E. Lamont, M. Ferrera, L. Razzari, F. Légaré, R. Morandotti, S. Chu, B. E. Little, and D. J. Moss, "Supercontinuum generation in a high index doped silica glass spiral waveguide," Optics Express, vol. 18, no. 2, pp. 923-930, 2010/01/18, 2010.

[23] A. Pasquazi, M. Peccianti, L. Razzari, D. J. Moss, S. Coen, M. Erkintalo, Y. K. Chembo, T. Hansson, S. Wabnitz, P. Del'Haye, X. Xue, A. M. Weiner, and R. Morandotti, "Micro-combs: A novel generation of optical sources," Physics Reports, vol. 729, pp. 1-81, 2018/01/27/, 2018.

[24] P. Del'Haye, A. Schliesser, O. Arcizet, T. Wilken, R. Holzwarth, and T. J. Kippenberg, “Optical frequency comb generation from a monolithic microresonator," Nature, vol. 450, no. 7173, pp. 1214-1217, 2007/12/01, 2007.

[25] T. J. Kippenberg, R. Holzwarth, and S. A. Diddams, "Microresonator-Based Optical Frequency Combs," Science, vol. 332, no. 6029, pp. 555-559, 2011.

[26] J. S. Levy, A. Gondarenko, M. A. Foster, A. C. Turner-Foster, A. L. Gaeta, and M. Lipson, "CMOS-compatible multiple-wavelength oscillator for on-chip optical interconnects," Nature Photonics, vol. 4, no. 1, pp. 37-40, 2010/01/01, 2010.

[27] W. Wang, Z. Lu, W. Zhang, S. T. Chu, B. E. Little, L. Wang, X. Xie, M. Liu, Q. Yang, L. Wang, J. Zhao, G. Wang, Q. Sun, Y. Liu, Y. Wang, and W. Zhao, "Robust soliton crystals in a thermally controlled microresonator," Optics Letters, vol. 43, no. 9, pp. 2002-2005, 2018/05/01, 2018.

[28] D. C. Cole, E. S. Lamb, P. Del'Haye, S. A. Diddams, and S. B. Papp, "Soliton crystals in Kerr resonators," Nature Photonics, vol. 11, no. 10, pp. 671-676, 2017/10/01, 2017.

[29] M. Karpov, M. H. P. Pfeiffer, H. Guo, W. Weng, J. Liu, and T. J. Kippenberg, "Dynamics of soliton crystals in optical microresonators," Nature Physics, vol. 15, no. 10, pp. 1071-1077, 2019/10/01, 2019.

[30] D. J. Moss, R. Morandotti, A. L. Gaeta, and M. Lipson, "New CMOS-compatible platforms based on silicon nitride and Hydex for nonlinear optics," Nature Photonics, vol. 7, no. 8, pp. 597-607, 2013/08/01, 2013. 
[31] M. Ferrera, L. Razzari, D. Duchesne, R. Morandotti, Z. Yang, M. Liscidini, J. E. Sipe, S. Chu, B. E. Little, and D. J. Moss, "Low-power continuous-wave nonlinear optics in doped silica glass integrated waveguide structures," Nature Photonics, vol. 2, no. 12, pp. 737-740, 2008/12/01, 2008.

[32] H. Bao, A. Cooper, M. Rowley, L. Di Lauro, J. S. Totero Gongora, S. T. Chu, B. E. Little, G.-L. Oppo, R. Morandotti, D. J. Moss, B. Wetzel, M. Peccianti, and A. Pasquazi, "Laser cavity-soliton microcombs," Nature Photonics, vol. 13, no. 6, pp. 384-389, 2019/06/01, 2019.

[33] A. Pasquazi, M. Peccianti, B. E. Little, S. T. Chu, D. J. Moss, and R. Morandotti, "Stable, dual mode, high repetition rate mode-locked laser based on a microring resonator," Optics Express, vol. 20, no. 24, pp. 27355 27363, 2012/11/19, 2012.

[34] A. Pasquazi, L. Caspani, M. Peccianti, M. Clerici, M. Ferrera, L. Razzari, D. Duchesne, B. E. Little, S. T. Chu, D. J. Moss, and R. Morandotti, "Self-locked optical parametric oscillation in a CMOS compatible microring resonator: a route to robust optical frequency comb generation on a chip," Optics Express, vol. 21, no. 11, pp. 13333-13341, 2013/06/03, 2013.

[35] A. Pasquazi, Y. Park, J. Azaña, F. Légaré, R. Morandotti, B. E. Little, S. T. Chu, and D. J. Moss, “Efficient wavelength conversion and net parametric gain via Four Wave Mixing in a high index doped silica waveguide," Optics Express, vol. 18, no. 8, pp. 7634-7641, 2010/04/12, 2010.

[36] V. Torres-Company, and A. M. Weiner, "Optical frequency comb technology for ultra-broadband radio-frequency photonics," Laser \& Photonics Reviews, vol. 8, no. 3, pp. 368-393, 2014.

[37] M. Peccianti, A. Pasquazi, Y. Park, B. E. Little, S. T. Chu, D. J. Moss, and R. Morandotti, "Demonstration of a stable ultrafast laser based on a nonlinear microcavity," Nature Communications, vol. 3, no. 1, pp. 765, 2012/04/03, 2012.

[38] L. Di Lauro, J. Li, D. J. Moss, R. Morandotti, S. T. Chu, M. Peccianti, and A. Pasquazi, "Parametric control of thermal self-pulsation in micro-cavities," Optics Letters, vol. 42, no. 17, pp. 3407-3410, 2017/09/01, 2017.

[39] H. Bao, A. Cooper, S. T. Chu, D. J. Moss, R. Morandotti, B. E. Little, M. Peccianti, and A. Pasquazi, "Type-II micro-comb generation in a filter-driven four wave mixing laser [Invited]," Photonics Research, vol. 6, no. 5, pp. B67-B73, 2018/05/01, 2018.

[40] Chou, J., Han, Y., and Jalali, B.: ‘Adaptive RF-photonic arbitrary waveform generator', IEEE Photonics Technology Letters, 2003, 15, (4), pp. 581-583

[41] X. Xue, Y. Xuan, H.-J. Kim, J. Wang, D. E. Leaird, M. Qi, and A. M. Weiner, "Programmable Single-Bandpass Photonic RF Filter Based on Kerr Comb from a Microring," Journal of Lightwave Technology, vol. 32, no. 20, pp. $3557-3565,2014 / 10 / 15,2014$.

[42] X. Xu, J. Wu, M. Shoeiby, T. G. Nguyen, S. T. Chu, B. E. Little, R. Morandotti, A. Mitchell, and D. J. Moss, "Reconfigurable broadband microwave photonic intensity differentiator based on an integrated optical frequency comb source," APL Photonics, vol. 2, no. 9, pp. 096104, 2017.

[43] X. Xu, M. Tan, J. Wu, R. Morandotti, A. Mitchell, and D. J. Moss, "Microcomb-Based Photonic RF Signal Processing," IEEE Photonics Technology Letters, vol. 31, no. 23, pp. 1854-1857, 2019.

[44] X. Xu, J. Wu, T. G. Nguyen, M. Shoeiby, S. T. Chu, B. E. Little, R. Morandotti, A. Mitchell, and D. J. Moss, "Advanced RF and microwave functions based on an integrated optical frequency comb source," Optics Express, vol. 26, no. 3, pp. 2569-2583, 2018/02/05, 2018.

[45] X. Xue, Y. Xuan, C. Bao, S. Li, X. Zheng, B. Zhou, M. Qi, and A. M. Weiner, "Microcomb-Based True-TimeDelay Network for Microwave Beamforming With Arbitrary Beam Pattern Control," Journal of Lightwave Technology, vol. 36, no. 12, pp. 2312-2321, 2018.

[46] J. Wu, X. Xu, T. G. Nguyen, S. T. Chu, B. E. Little, R. Morandotti, A. Mitchell, and D. J. Moss, "RF Photonics: An Optical Microcombs' Perspective," IEEE Journal of Selected Topics in Quantum Electronics, vol. 24, no. 4, pp. 120, 2018.

[47] X. Xu, J. Wu, T. G. Nguyen, S. T. Chu, B. E. Little, R. Morandotti, A. Mitchell, and D. J. Moss, "Broadband RF Channelizer Based on an Integrated Optical Frequency Kerr Comb Source," Journal of Lightwave Technology, vol. 36, no. 19, pp. 4519-4526, 2018.

[48] X. Xu, J. Wu, M. Tan, T. G. Nguyen, S. T. Chu, B. E. Little, R. Morandotti, A. Mitchell, and D. J. Moss, "Orthogonally Polarized RF Optical Single Sideband Generation and Dual-Channel Equalization Based on an Integrated Microring Resonator," Journal of Lightwave Technology, vol. 36, no. 20, pp. 4808-4818, 2018.

[49] Y. Zhang, X. Xu, J. Wu, L. Jia, M. Tan, T. Nguyen, S. T. Chu, B. Little, R. Morandotti, A. Mitchell, and D. Moss, Continuously tunable orthogonally polarized RF optical single sideband generator based on cascaded micro-ring resonators, p.^pp. AU: SPIE, 2019. 
[50] X. Xu, J. Wu, T. G. Nguyen, T. Moein, S. T. Chu, B. E. Little, R. Morandotti, A. Mitchell, and D. J. Moss, "Photonic microwave true time delays for phased array antennas using a 49\&\#x2009;\&\#x2009;GHz FSR integrated optical micro-comb source [Invited]," Photonics Research, vol. 6, no. 5, pp. B30-B36, 2018/05/01, 2018.

[51] X. Xu, M. Tan, J. Wu, T. G. Nguyen, S. T. Chu, B. E. Little, R. Morandotti, A. Mitchell, and D. J. Moss, "Advanced Adaptive Photonic RF Filters with 80 Taps Based on an Integrated Optical Micro-Comb Source," Journal of Lightwave Technology, vol. 37, no. 4, pp. 1288-1295, 2019/02/15, 2019.

[52] W. Liang, D. Eliyahu, V. S. Ilchenko, A. A. Savchenkov, A. B. Matsko, D. Seidel, and L. Maleki, "High spectral purity Kerr frequency comb radio frequency photonic oscillator," Nature Communications, vol. 6, no. 1, pp. 7957, 2015/08/11, 2015.

[53] J. Liu, E. Lucas, A. S. Raja, J. He, J. Riemensberger, R. N. Wang, M. Karpov, H. Guo, R. Bouchand, and T. J. Kippenberg, "Photonic microwave generation in the X- and K-band using integrated soliton microcombs," Nature Photonics, vol. 14, no. 8, pp. 486-491, 2020/08/01, 2020.

[54] M. Tan, X. Xu, J. Wu, R. Morandotti, A. Mitchell, and D. J. Moss, "Photonic RF and microwave filters based on $49 \mathrm{GHz}$ and $200 \mathrm{GHz}$ Kerr microcombs," Optics Communications, vol. 465, pp. 125563, 2020/06/15/, 2020.

[55] X. Xu, M. Tan, J. Wu, A. Boes, T. G. Nguyen, S. T. Chu, B. E. Little, R. Morandotti, A. Mitchell, and D. J. Moss, "Broadband Photonic RF Channelizer With 92 Channels Based on a Soliton Crystal Microcomb," Journal of Lightwave Technology, vol. 38, no. 18, pp. 5116-5121, 2020.

[56] X. Xu, M. Tan, J. Wu, A. Boes, B. Corcoran, T. G. Nguyen, S. T. Chu, B. E. Little, R. Morandotti, A. Mitchell, and D. Moss, "Photonic RF Phase-Encoded Signal Generation With a Microcomb Source," Journal of Lightwave Technology, vol. 38, no. 7, pp. 1722-1727, 2020.

[57] X. Xu, M. Tan, J. Wu, T. G. Nguyen, S. T. Chu, B. E. Little, R. Morandotti, A. Mitchell, and D. J. Moss, "High performance RF filters via bandwidth scaling with Kerr micro-combs," APL Photonics, vol. 4, no. 2, pp. 026102 2019.

[58] M. Tan, X. Xu, B. Corcoran, J. Wu, A. Boes, T. G. Nguyen, S. T. Chu, B. E. Little, R. Morandotti, A. Mitchell, and D. J. Moss, "Microwave and RF Photonic Fractional Hilbert Transformer Based on a 50 GHz Kerr Micro-Comb," Journal of Lightwave Technology, vol. 37, no. 24, pp. 6097-6104, 2019.

[59] M. Tan, X. Xu, B. Corcoran, J. Wu, A. Boes, T. G. Nguyen, S. T. Chu, B. E. Little, R. Morandotti, A. Mitchell, and D. J. Moss, "RF and Microwave Fractional Differentiator Based on Photonics," IEEE Transactions on Circuits and Systems II: Express Briefs, vol. 67, no. 11, pp. 2767-2771, 2020.

[60] M. Tan, X. Xu, A. Boes, B. Corcoran, J. Wu, T. G. Nguyen, S. T. Chu, B. E. Little, R. Morandotti, A. Mitchell, and D. J. Moss, "Photonic RF Arbitrary Waveform Generator Based on a Soliton Crystal Micro-Comb Source," Journal of Lightwave Technology, vol. 38, no. 22, pp. 6221-6226, 2020/11/15, 2020.

[61] M. Tan, X. Xu, J. Wu, R. Morandotti, A. Mitchell, and D. Moss, "RF and microwave photonic temporal signal processing with Kerr micro-combs," Advances in Physics: X, vol. 6, no. 1, pp. 1-46, 2021.

[62] J. Wu, X. Xu, T. G. Nguyen, S. T. Chu, B. E. Little, R. Morandotti, A. Mitchell, and D. J. Moss, "Orthogonally polarized optical single sideband generation based on integrated microring resonators," OSA Technical Digest. $\mathrm{p}$. M1B.5.

[63] Mengxi Tan, Xingyuan Xu, Jiayang Wu, Bill Corcoran, Andreas Boes, Thach G. Nguyen, Sai T. Chu, Brent E. Little, Roberto Morandotti, Arnan Mitchell, and David J. Moss, "Integral order photonic RF signal processors based on a soliton crystal micro-comb source", IOP Journal of Optics Vol. 23 (11) 125701 (2021).

[64] Y. Park, M. H. Asghari, R. Helsten, and J. Azana, "Implementation of Broadband Microwave Arbitrary-Order Time Differential Operators Using a Reconfigurable Incoherent Photonic Processor," IEEE Photonics Journal, vol. 2, no. 6, pp. 1040-1050, 2010.

[65] X. Xu, M. Tan, B. Corcoran, J. Wu, T. G. Nguyen, A. Boes, S. T. Chu, B. E. Little, R. Morandotti, A. Mitchell, D. G. Hicks, and D. J. Moss, "Photonic Perceptron Based on a Kerr Microcomb for High-Speed, Scalable, Optical Neural Networks," Laser \& Photonics Reviews, vol. 14, no. 10, pp. 2000070, 2020.

[66] X. Xu, M. Tan, B. Corcoran, J. Wu, A. Boes, T. G. Nguyen, S. T. Chu, B. E. Little, D. G. Hicks, R. Morandotti, A. Mitchell, and D. J. Moss, "11 TOPS photonic convolutional accelerator for optical neural networks," Nature, vol. 589, no. 7840, pp. 44-51, 2021/01/01, 2021.

[67] J. Feldmann, N. Youngblood, M. Karpov, H. Gehring, X. Li, M. Stappers, M. Le Gallo, X. Fu, A. Lukashchuk, A. S. Raja, J. Liu, C. D. Wright, A. Sebastian, T. J. Kippenberg, W. H. P. Pernice, and H. Bhaskaran, "Parallel convolutional processing using an integrated photonic tensor core," Nature, vol. 589, no. 7840, pp. 52-58, 2021/01/01, 2021. 
[68] X. Xu, M. Tan, J. Wu, T. Nguyen, S. Chu, B. Little, R. Morandotti, A. Mitchell, and D. Moss, "High performance RF filters via bandwidth scaling with Kerr micro-combs," APL Photonics, vol. 4, no. 2, pp. 1-8, 2019.

[69] D. Marpaung, J. Yao, and J. Capmany, “Integrated microwave photonics," Nature Photonics, vol. 13, no. 2, pp. 8090, 2019/02/01, 2019.

[70] Z. Zhu, H. Chi, T. Jin, S. Zheng, X. Jin, and X. Zhang, “All-positive-coefficient microwave photonic filter with rectangular response," Optics Letters, vol. 42, no. 15, pp. 3012-3015, 2017/08/01, 2017.

[71] R. A. Minasian, "Ultra-Wideband and Adaptive Photonic Signal Processing of Microwave Signals," IEEE Journal of Quantum Electronics, vol. 52, no. 1, pp. 1-13, 2016.

[72] W. Wang, W. Zhang, Z. Lu, S. T. Chu, B. E. Little, Q. Yang, L. Wang, and W. Zhao, "Self-locked orthogonal polarized dual comb in a microresonator," Photonics Research, vol. 6, no. 5, pp. 363-367, 2018/05/01, 2018.

[73] J. Capmany, J. Mora, I. Gasulla, J. Sancho, J. Lloret, and S. Sales, "Microwave Photonic Signal Processing," Journal of Lightwave Technology, vol. 31, no. 4, pp. 571-586, 2013/02/15, 2013.

[74] R. A. Minasian, "Photonic signal processing of microwave signals," IEEE Transactions on Microwave Theory and Techniques, vol. 54, no. 2, pp. 832-846, 2006.

[75] J. Capmany, and D. Novak, "Microwave photonics combines two worlds," Nature Photonics, vol. 1, no. 6, pp. 319330, 2007/06/01, 2007.

[76] R. Williamson, and R. D. Esman, "RF Photonics,” Journal of Lightwave Technology, vol. 26, no. 9, pp. 1145-1153, 2008/05/01, 2008.

[77] Y. Liu, J. Hotten, A. Choudhary, B. J. Eggleton, and D. Marpaung, “All-optimized integrated RF photonic notch filter," Optics Letters, vol. 42, no. 22, pp. 4631-4634, 2017/11/15, 2017.

[78] C. Tseng, and S.-C. Pei, "Design and application of discrete-time fractional Hilbert transformer," Circuits and Systems II: Analog and Digital Signal Processing, IEEE Transactions on, vol. 47, pp. 1529-1533, 01/01, 2001.

[79] L. Razzari, D. Duchesne, M. Ferrera, R. Morandotti, S. Chu, B. E. Little, and D. J. Moss, "CMOS-compatible integrated optical hyper-parametric oscillator," Nature Photonics, vol. 4, no. 1, pp. 41-45, 2010/01/01, 2010.

[80] X. Xu, J. Wu, M. Tan, T. G. Nguyen, S. T. Chu, B. E. Little, R. Morandotti, A. Mitchell, and D. J. Moss, "Broadband Microwave Frequency Conversion Based on an Integrated Optical Micro-Comb Source," Journal of Lightwave Technology, vol. 38, no. 2, pp. 332-338, 2020.

[81] J. A. Davis, D. E. McNamara, and D. M. Cottrell, “Analysis of the fractional Hilbert transform," Appl. Opt., vol. 37, no. 29, pp. 6911-6913, Oct. 1998 .

[82] C. D. Holdenried, J. W. Haslett, and B. Davies, “A fully integrated 10-Gb/s tapped delay Hilbert transformer for optical single sideband," IEEE Microw. Wireless Compon. Lett., vol. 15, no. 5, pp. 303-305, May 2005.

[83] H. Emami, N. Sarkhosh, L. A. Bui, and A. Mitchell, "Wideband RF photonic in-phase and quadrature-phase generation," Opt. Lett., vol. 33, no. 2, pp. 98-100, Jan 15. 2008.

[84] W. Liu, et al., "A Fully Reconfigurable Photonic Integrated Signal Processor," Nature Photonics, vol. 10, no. 3, pp. 190-196, 2016.

[85] F. Zeng, and J. Yao, “An Approach to Ultrawideband Pulse Generation and Distribution Over Optical Fiber,” IEEE Photonics Technol. Lett., vol. 18, no. 7, pp. 823-825, Apr. 2006.

[86] S. Pan, and J. Yao, "Optical generation of polarity- and shape-switchable ultrawideband pulses using a chirped intensity modulator and a first-order asymmetric Mach-Zehnder interferometer," Opt. Lett., vol. 34, no. 9, pp. 13121314, May. 2009.

[87] Y. Yu, J. Dong, X. Li, and X. Zhang, "Ultra-Wideband Generation Based on Cascaded Mach-Zehnder Modulators," IEEE Photonics Technol. Lett., vol. 23, no. 23, Dec. 2011.

[88] A. O.-Blanch, J. Mora, J. Capmany, B. Ortega, and D. Pastor, "Tunable radio-frequency photonic filter based on an actively mode-locked fiber laser," Opt. Lett., vol. 31, no. 6, pp. 709-711, Mar. 2006.

[89] V. R. Supradeepa, C. M. Long, R. Wu. F. Ferdous, E. Hamidi, D. E. Leaird, and A. M. Weiner, "Comb-based radiofrequency photonic filters with rapid tunability and high selectivity," Nature Photonics, vol. 6, pp. 186-194, Mar. 2012.

[90] V. T.-Company, and A. M. Weiner, "Optical frequency comb technology for ultra-broadband radio-frequency photonics," Laser Photonics Rev., vol. 8, no. 3, pp. 368-393, 2014.

[91] Ghelfi, P., Laghezza, F., Scotti, F., Serafino, G., Capria, A., Pinna, S., Onori, D., Porzi, C., Scaffardi, M., Malacarne, A., Vercesi, V., Lazzeri, E., Berizzi, F., and Bogoni, A.: ‘A fully photonics-based coherent radar system', Nature, 2014, 507, (7492), pp. 341-345 
[92] Skolnik, M.: 'Role of radar in microwaves', IEEE Transactions on Microwave Theory and Techniques, 2002, 50, (3), pp. 625-632

[93] Cundiff, S.T., and Weiner, A.M.: 'Optical arbitrary waveform generation', Nat. Photonics, 2010, 4, (11), pp. 760-766

[94] Rashidinejad, A., Li, Y., and Weiner, A.M.: 'Recent Advances in Programmable Photonic-Assisted Ultrabroadband Radio-Frequency Arbitrary Waveform Generation', IEEE Journal of Quantum Electronics, 2016, 52, (1), pp. 1-17

[95] Ghelfi, P., Scotti, F., Laghezza, F., and Bogoni, A.: 'Photonic Generation of Phase-Modulated RF Signals for Pulse Compression Techniques in Coherent Radars', Journal of Lightwave Technology, 2012, 30, (11), pp. 1638-1644

[96] Khan, M.H., Shen, H., Xuan, Y., Zhao, L., Xiao, S., Leaird, D.E., Weiner, A.M., and Qi, M.: 'Ultrabroad-bandwidth arbitrary radiofrequency waveform generation with a silicon photonic chip-based spectral shaper', Nat. Photonics, 2010, 4, (2), pp. 117-122

[97] Chi, H., and Yao, J.: 'Photonic Generation of Phase-Coded Millimeter-Wave Signal Using a Polarization Modulator', IEEE Microwave and Wireless Components Letters, 2008, 18, (5), pp. 371-373

[98] Zhang, Y., and Pan, S.: 'Generation of phase-coded microwave signals using a polarization-modulator-based photonic microwave phase shifter', Opt. Lett., 2013, 38, (5), pp. 766-768

[99] Zhu, S., Shi, Z., Li, M., Zhu, N.H., and Li, W.: 'Simultaneous frequency upconversion and phase coding of a radiofrequency signal for photonic radars', Opt. Lett., 2018, 43, (3), pp. 583-586

[100] Zhu, S., Li, M., Wang, X., Zhu, N.H., Cao, Z.Z., and Li, W.: 'Photonic generation of background-free binary phasecoded microwave pulses', Opt. Lett., 2019, 44, (1), pp. 94-97

[101] Li, Z., Li, W., Chi, H., Zhang, X., and Yao, J.: 'Photonic generation of phase-coded microwave signal with large frequency tunability', IEEE Photonics Technology Letters, 2011, 23, (11), pp. 712-714

[102] Liu, W., and Yao, J.: 'Photonic generation of microwave waveforms based on a polarization modulator in a Sagnac loop', Journal of Lightwave Technology, 2014, 32, (20), pp. 3637-3644

[103] Wang, J., Shen, H., Fan, L., Wu, R., Niu, B., Varghese, L.T., Xuan, Y., Leaird, D.E., Wang, X., and Gan, F.: 'Reconfigurable radio-frequency arbitrary waveforms synthesized in a silicon photonic chip', Nat. Commun., 2015, $6,(1)$, pp. 1-8

[104] Ashrafi, R., Li, M., and Azaña, J.: 'Multi-TBaud optical coding based on superluminal space-to-time mapping in long period gratings', Optics and Photonics Journal, 2013, 3, (2), pp. 126-130

[105] Rashidinejad, A., and Weiner, A.M.: 'Photonic radio-frequency arbitrary waveform generation with maximal timebandwidth product capability', Journal of Lightwave Technology, 2014, 32, (20), pp. 3383-3393

[106] Jiang, Z., Huang, C.-B., Leaird, D.E., and Weiner, A.M.: 'Optical arbitrary waveform processing of more than 100 spectral comb lines', Nat. Photonics, 2007, 1, (8), pp. 463-467

[107] Dai, Y., and Yao, J.: 'Microwave pulse phase encoding using a photonic microwave delay-line filter', Opt. Lett., 2007, 32, (24), pp. 3486-3488

[108] S. Pan, J. Yao, "Optical generation of polarity- and shape-switchable ultrawideband pulses using a chirped intensity modulator and a first-order asymmetric Mach-Zehnder interferometer," Opt. Lett., vol. 34, no. 9, pp. 1312-1314, 2009.

[109] X. Li, J. Dong, Y. Yu, and X. Zhang, "A Tunable Microwave Photonic Filter Based on an All-Optical Differentiator," IEEE Photon. Technol. Lett., vol. 23, no. 22, pp. 308-310, Mar. 2011.

[110] Y. Han, Z. Li, and J. Yao, “A Microwave Bandpass Differentiator Implemented Based on a Nonuniformly-Spaced Photonic Microwave Delay-Line Filter,” J. Lightw. Technol., vol. 29, no. 22, pp. 3470-3475, Nov. 2011.

[111] R. Ashrafi and J. Azaña, "Figure of merit for photonic differentiators," Opt. Exp., vol. 20, no. 3, pp. 2626-2639, Jan. 2012.

[112] F. Zeng and J. Yao, "Ultrawideband Impulse Radio Signal Generation Using a High-Speed Electrooptic Phase Modulator and a Fiber-Bragg-Grating-Based Frequency Discriminator," IEEE Photon. Technol. Lett., vol. 18, no. 19, pp. 2062-2064, Oct. 2006.

[113] P. Li, H. Chen, M. Chen, and S. Xie, "Gigabit/s Photonic Generation, Modulation, and Transmission for a Reconfigurable Impulse Radio UWB Over Fiber System,” IEEE Photon. Technol. Lett., vol. 4, no. 3, pp. 805-816, Jun. 2012.

[114] Y. Yu, F. Jiang, H. Tang, L. Xu, X. Liu, J. Dong, and X. Zhang, "Reconfigurable photonic temporal differentiator based on a dual-drive Mach-Zehnder modulator," Opt. Exp., vol. 24, no. 11, pp. 11739-11748, May 2016.

[115] P. Velanas, A. Bogris, A. Argyris, and D. Syvridis, "High-Speed All-Optical First- and Second-Order Differentiators Based on Cross-Phase Modulation in Fibers," J. Lightw. Technol., vol. 26, no. 18, pp. 3269-3276, Sep. 2008.

[116] J. Xu, X. Zhang, J. Dong, D. Liu, and D. Huang, "All-optical differentiator based on cross-gain modulation in semiconductor optical amplifier," Opt. Lett., vol. 32, no. 20, pp. 3029-3031, Oct. 2007. 
[117] J. Xu, X. Zhang, J. Dong, D. Liu, and D. Huang, "High-speed all-optical differentiator based on a semiconductor optical amplifier and an optical filter," Opt. Lett., vol. 32, no. 13, pp. 1872-1874, Jul. 2007.

[118] F. Wang, J. Dong, E. Xu, and X. Zhang, "All-optical UWB generation and modulation using SOA-XPM effect and DWDM-based multi-channel frequency discrimination,” Opt. Exp., vol. 18, no. 24, pp. 24588-24594, Nov. 2010.

[119] V. Moreno, M. Rius, J. Mora, M. A. Muriel, and J. Capmany, "Integrable high order UWB pulse photonic generator based on cross phase modulation in a SOA-MZI," Opt. Exp., vol. 21, no. 19, pp. 22911-22917, Sep. 2013.

[120] Q. Wang and J. Yao, "Switchable optical UWB monocycle and doublet generation using a reconfigurable photonic microwave delay-line filter," Opt. Exp., vol. 15, no. 22, pp. 14667-14672, Oct. 2007.

[121] M. Bolea, J. Mora, B. Ortega, and J. Capmany, "Optical UWB pulse generator using an $N$ tap microwave photonic filter and phase inversion adaptable to different pulse modulation formats," Opt. Exp., vol. 17, no. 7, pp. 5023-50332, Mar. 2009.

[122] B. Mathieu, P. Melchior, A. Oustaloup, C. Ceyral, "Fractional differentiation for edge detection," Signal Processing, vol. 83, pp. 2421-2432, Nov. 2003.

[123] A. Oustaloup, F. Levron, B. Mathieu, and F. M. Nanot, "Frequency-Band Complex Noninteger Differentiator: Characterization and Synthesis," IEEE Trans. on Circuit and Systems - I: Fundamental Theory and Application, vol. 47, no. 1, pp. 25-39, Jan. 2000.

[124] F. Li, Y. Park, and J. Azaña, "Linear Characterization of Optical Pulses With Durations Ranging From the Picosecond to the Nanosecond Regime Using Ultrafast Photonic Differentiation,” J. Lightw. Technol., vol. 27, no. 1, pp. 46234633, 2009.

[125] M. Tan, X. Xu, J. Wu, T. G. Nguyen, S. T. Chu, B. E. Little, R. Morandotti, A. Mitchell, and D. J. Moss, "Photonic Radio Frequency Channelizers based on Kerr Optical Micro-combs", IOP Journal of Semiconductors Vol. 42 (4), 041302 (2021).

[126] M. Tan, X. Xu, J. Wu, T. G. Nguyen, S. T. Chu, B. E. Little, R. Morandotti, A. Mitchell, and D. J. Moss, "Orthogonally polarized Photonic Radio Frequency single sideband generation with integrated micro-ring resonators", IOP Journal of Semiconductors, Vol. 42 (4), 041305 (2021).

[127] C.Prayoonyong et al., "Frequency comb distillation for optical superchannel transmission", Journal of Lightwave Technology Vol. 39 (23) 7383-7392 (2021).

[128] X. Xu et al, "Photonic perceptron based on a Kerr microcomb for scalable high speed optical neural networks", Laser and Photonics Reviews, vol. 14, no. 8, 2000070 (2020). DOI: 10.1002/lpor.202000070.

[129] X. Xu, et al., "11 TOPs photonic convolutional accelerator for optical neural networks", Nature 589, 44-51 (2021).

[130] Pasquazi, A. et al. Micro-combs: a novel generation of optical sources. Physics Reports 729, 1-81 (2018).

[131] Moss, D. J. et al., "New CMOS-compatible platforms based on silicon nitride and Hydex for nonlinear optics", Nature photonics 7, 597 (2013).

[132] B. Corcoran, et al., "Ultra-dense optical data transmission over standard fiber with a single chip source", Nature Communications, vol. 11, Article:2568, 2020.

[133] Kues, M. et al. Quantum optical microcombs. Nature Photonics 13, (3) 170-179 (2019). doi:10.1038/s41566-0190363-0

[134] C. Reimer, L. Caspani, M. Clerici, et al., "Integrated frequency comb source of heralded single photons," Optics Express, vol. 22, no. 6, pp. 6535-6546, 2014.

[135] C. Reimer, et al., "Cross-polarized photon-pair generation and bi-chromatically pumped optical parametric oscillation on a chip", Nature Communications, vol. 6, Article 8236, 2015. DOI: 10.1038/ncomms9236.

[136] L. Caspani, C. Reimer, M. Kues, et al., "Multifrequency sources of quantum correlated photon pairs on-chip: a path toward integrated Quantum Frequency Combs," Nanophotonics, vol. 5, no. 2, pp. 351-362, 2016.

[137] C. Reimer et al., "Generation of multiphoton entangled quantum states by means of integrated frequency combs," Science, vol. 351, no. 6278, pp. 1176-1180, 2016.

[138] M. Kues, et al., "On-chip generation of high-dimensional entangled quantum states and their coherent control", Nature, vol. 546, no. 7660, pp. 622-626, 2017.

[139] P. Roztocki et al., "Practical system for the generation of pulsed quantum frequency combs," Optics Express, vol. 25, no. 16, pp. 18940-18949, 2017.

[140] Y. Zhang, et al., "Induced photon correlations through superposition of two four-wave mixing processes in integrated cavities", Laser and Photonics Reviews, vol. 14, no. 7, pp. 2000128, 2020. DOI: 10.1002/lpor.202000128

[141] C. Reimer, et al.,"High-dimensional one-way quantum processing implemented on d-level cluster states", Nature Physics, vol. 15, no.2, pp. 148-153, 2019. 
[142] Stern, B., Ji, X., Okawachi, Y., Gaeta, A. L. \& Lipson, M. Battery-operated integrated frequency comb generator. Nature 562, 401 (2018).

[143] H. Bao, et al., Laser cavity-soliton microcombs, Nature Photonics, vol. 13, no. 6, pp. 384-389, Jun. 2019.

[144] Bao, C., et al., Direct soliton generation in microresonators, Opt. Lett, 42, 2519 (2017).

[145] M.Ferrera et al., "CMOS compatible integrated all-optical RF spectrum analyzer", Optics Express, vol. 22, no. 18, 21488 - 21498 (2014).

[146] A. Pasquazi, et al., "Sub-picosecond phase-sensitive optical pulse characterization on a chip", Nature Photonics, vol. 5, no. 10, pp. 618-623 (2011).

[147] M. Kues, et al., "Passively modelocked laser with an ultra-narrow spectral width", Nature Photonics, vol. 11, no. 3, pp. 159, 2017.

[148] L. Razzari, et al., "CMOS-compatible integrated optical hyper-parametric oscillator," Nature Photonics, vol. 4, no. 1 , pp. 41-45, 2010.

[149] M. Ferrera, et al., "Low-power continuous-wave nonlinear optics in doped silica glass integrated waveguide structures," Nature Photonics, vol. 2, no. 12, pp. 737-740, 2008.

[150] M.Ferrera et al.“On-Chip ultra-fast 1st and 2nd order CMOS compatible all-optical integration”, Opt. Express, vol. 19, (23)pp. 23153-23161 (2011).

[151] D. Duchesne, M. Peccianti, M. R. E. Lamont, et al., "Supercontinuum generation in a high index doped silica glass spiral waveguide," Optics Express, vol. 18, no, 2, pp. 923-930, 2010.

[152] H Bao, L Olivieri, M Rowley, ST Chu, BE Little, R Morandotti, DJ Moss, ... "Turing patterns in a fiber laser with a nested microresonator: Robust and controllable microcomb generation”, Physical Review Research 2 (2), 023395 (2020).

[153] M. Ferrera, et al., "On-chip CMOS-compatible all-optical integrator”, Nature Communications, vol. 1, Article 29, 2010.

[154] A. Pasquazi, et al., "All-optical wavelength conversion in an integrated ring resonator," Optics Express, vol. 18 , no. 4, pp. 3858-3863, 2010.

[155] A. Pasquazi, Y. Park, J. Azana, et al., "Efficient wavelength conversion and net parametric gain via Four Wave Mixing in a high index doped silica waveguide," Optics Express, vol. 18, no. 8, pp. 7634-7641, 2010.

[156] M. Peccianti, M. Ferrera, L. Razzari, et al., "Subpicosecond optical pulse compression via an integrated nonlinear chirper," Optics Express, vol. 18, no. 8, pp. 7625-7633, 2010.

[157] Little, B. E. et al., "Very high-order microring resonator filters for WDM applications", IEEE Photonics Technol. Lett. 16, 2263-2265 (2004).

[158] M. Ferrera et al., "Low Power CW Parametric Mixing in a Low Dispersion High Index Doped Silica Glass MicroRing Resonator with Q-factor > 1 Million", Optics Express, vol.17, no. 16, pp. 14098-14103 (2009).

[159] M. Peccianti, et al., "Demonstration of an ultrafast nonlinear microcavity modelocked laser", Nature Communications, vol. 3, pp. 765, 2012.

[160] A. Pasquazi, et al., "Self-locked optical parametric oscillation in a CMOS compatible microring resonator: a route to robust optical frequency comb generation on a chip," Optics Express, vol. 21, no. 11, pp. 13333-13341, 2013.

[161] A. Pasquazi, et al., "Stable, dual mode, high repetition rate mode-locked laser based on a microring resonator," Optics Express, vol. 20, no. 24, pp. 27355-27362, 2012.

[162] Wu, J. et al. RF Photonics: An Optical Microcombs' Perspective. IEEE Journal of Selected Topics in Quantum Electronics Vol. 24, 6101020, 1-20 (2018).

[163] Xu, X., et al., Photonic microwave true time delays for phased array antennas using a $49 \mathrm{GHz}$ FSR integrated microcomb source, Photonics Research, 6, B30-B36 (2018).

[164] T. G. Nguyen et al., "Integrated frequency comb source-based Hilbert transformer for wideband microwave photonic phase analysis," Opt. Express, vol. 23, no. 17, pp. 22087-22097, Aug. 2015.

[165] X. Xu, J. Wu, M. Shoeiby, T. G. Nguyen, S. T. Chu, B. E. Little, R. Morandotti, A. Mitchell, and D. J. Moss, "Reconfigurable broadband microwave photonic intensity differentiator based on an integrated optical frequency comb source," APL Photonics, vol. 2, no. 9, 096104, Sep. 2017.

[166] X. Xu, M. Tan, J. Wu, R. Morandotti, A. Mitchell, and D. J. Moss, "Microcomb-based photonic RF signal processing”, IEEE Photonics Technology Letters, vol. 31 no. 23 1854-1857, 2019.

[167] X. Xu, et al., "Broadband RF channelizer based on an integrated optical frequency Kerr comb source," Journal of Lightwave Technology, vol. 36, no. 19, pp. 4519-4526, 2018.

[168] X. Xu, et al., "Continuously tunable orthogonally polarized RF optical single sideband generator based on microring resonators," Journal of Optics, vol. 20, no. 11, 115701. 2018. 
[169] X. Xu, et al., "Orthogonally polarized RF optical single sideband generation and dual-channel equalization based on an integrated microring resonator," Journal of Lightwave Technology, vol. 36, no. 20, pp. 4808-4818. 2018.

[170] M.Tan, X. Xu, J. Wu, B. Corcoran, A. Boes, T. G. Nguyen, Sai T. Chu, B. E. Little, R.Morandotti, A. Mitchell, and D. J. Moss, "Integral order photonic RF signal processors based on a soliton crystal micro-comb source", IOP Journal of Optics vol. 23 (11) 125701 (2021).

[171] X. Xu, et al., "Advanced adaptive photonic RF filters with 80 taps based on an integrated optical micro-comb source," Journal of Lightwave Technology, vol. 37, no. 4, pp. 1288-1295, 2019.

[172] X. Xu, et al., Broadband microwave frequency conversion based on an integrated optical micro-comb source", Journal of Lightwave Technology, vol. 38 no. 2, pp. 332-338, 2020.

[173] M. Tan, et al., "Photonic RF and microwave filters based on 49GHz and 200GHz Kerr microcombs", Optics Comm. vol. 465,125563, Feb. 22. 2020.

[174] X. Xu, et al., "Broadband photonic RF channelizer with 90 channels based on a soliton crystal microcomb", Journal of Lightwave Technology, Vol. 38, no. 18, pp. 5116 - 5121, 2020. doi: 10.1109/JLT.2020.2997699.

[175] X. Xu, et al., "Photonic RF and microwave integrator with soliton crystal microcombs", IEEE Transactions on Circuits and Systems II: Express Briefs, vol. 67, no. 12, pp. 3582-3586, 2020. DOI:10.1109/TCSII.2020.2995682.

[176] X. Xu, et al., "Photonic RF phase-encoded signal generation with a microcomb source", J. Lightwave Technology, vol. 38, no. 7, 1722-1727, 2020.

[177] X. Xu, et al., "High performance RF filters via bandwidth scaling with Kerr micro-combs," APL Photonics, vol. 4, no. 2, pp. 026102. 2019.

[178] M. Tan, et al., "Microwave and RF photonic fractional Hilbert transformer based on a 50 GHz Kerr micro-comb", Journal of Lightwave Technology, vol. 37, no. 24, pp. 6097 - 6104, 2019.

[179] M. Tan, et al., "RF and microwave fractional differentiator based on photonics", IEEE Transactions on Circuits and Systems: Express Briefs, vol. 67, no.11, pp. 2767-2771, 2020. DOI:10.1109/TCSII.2020.2965158.

[180] M. Tan, et al., "Photonic RF arbitrary waveform generator based on a soliton crystal micro-comb source", Journal of Lightwave Technology, vol. 38, no. 22, pp. 6221-6226, Oct 22. 2020. DOI: 10.1109/JLT.2020.3009655.

[181] M. Tan, X. Xu, J. Wu, R. Morandotti, A. Mitchell, and D. J. Moss, "RF and microwave high bandwidth signal processing based on Kerr Micro-combs", Advances in Physics X, VOL. 6, NO. 1, 1838946 (2021). DOI:10.1080/23746149.2020.1838946.

[182] X. Xu, et al., "Advanced RF and microwave functions based on an integrated optical frequency comb source," Opt. Express, vol. 26 (3) 25692018.

[183] Kues, M. et al. Quantum optical microcombs. Nature Photonics 13, (3) 170-179 (2019). doi:10.1038/s41566-0190363-0

[184] P.Roztocki et al., "Complex quantum state generation and coherent control based on integrated frequency combs", Journal of Lightwave Technology 37 (2) 338-347 (2019).

[185] S. Sciara et al., "Generation and Processing of Complex Photon States with Quantum Frequency Combs", IEEE Photonics Technology Letters 31 (23) 1862-1865 (2019). DOI: 10.1109/LPT.2019.2944564.

[186] L. Caspani, C. Reimer, M. Kues, et al., "Multifrequency sources of quantum correlated photon pairs on-chip: a path toward integrated Quantum Frequency Combs," Nanophotonics, vol. 5, no. 2, pp. 351-362, 2016.

[187] C. Reimer et al., "Generation of multiphoton entangled quantum states by means of integrated frequency combs," Science, vol. 351, no. 6278, pp. 1176-1180, 2016.

[188] M. Kues, et al., "On-chip generation of high-dimensional entangled quantum states and their coherent control", Nature, vol. 546, no. 7660, pp. 622-626, 2017.

[189] P. Roztocki et al., "Practical system for the generation of pulsed quantum frequency combs," Optics Express, vol. 25, no.16, 18940-18949, 2017.

[190] Y. Zhang, et al., "Induced photon correlations through superposition of two four-wave mixing processes in integrated cavities", Laser and Photonics Reviews, vol. 14, no. 7, pp. 2000128, 2020. DOI: 10.1002/lpor.202000128

[191] C. Reimer, et al.,"High-dimensional one-way quantum processing implemented on d-level cluster states", Nature Physics, vol. 15 (2) 148 (2019).

[192] H. Bao, et al., Laser cavity-soliton microcombs, Nature Photonics, vol. 13, no. 6, pp. 384-389, Jun. 2019.

[193] Bao, C., et al., Direct soliton generation in microresonators, Opt. Lett, 42, 2519 (2017).

[194] M.Ferrera et al., "CMOS compatible integrated all-optical RF spectrum analyzer", Optics Express, vol. 22, (18) 21488 (2014).

[195] A. Pasquazi, et al., "Sub-picosecond phase-sensitive optical pulse characterization on a chip", Nature Photonics, vol. 5, no. 10, pp. 618-623 (2011). 
[196] M. Kues, et al., "Passively modelocked laser with an ultra-narrow spectral width", Nature Photonics, vol. 11, no. 3, pp. 159, 2017.

[197] L. Razzari, et al., "CMOS-compatible integrated optical hyper-parametric oscillator,” Nature Photonics, vol. 4 , no. $1,41-45,2010$.

[198] M. Ferrera, et al., "Low-power continuous-wave nonlinear optics in doped silica glass integrated waveguide structures," Nature Photonics, vol. 2, no. 12, pp. 737-740, 2008.

[199] M.Ferrera et al.“On-Chip ultra-fast 1st and 2nd order CMOS compatible all-optical integration”, Opt. Express, vol. 19, (23)pp. 23153-23161 (2011).

[200] D. Duchesne, M. Peccianti, M. R. E. Lamont, et al., "Supercontinuum generation in a high index doped silica glass spiral waveguide," Optics Express, vol. 18, no, 2, pp. 923-930, 2010.

[201] H Bao, L Olivieri, M Rowley, ST Chu, BE Little, R Morandotti, DJ Moss, ... “Turing patterns in a fiber laser with a nested microresonator: Robust and controllable microcomb generation”, Physical Review Research 2 (2), 023395 (2020).

[202] M. Ferrera, et al., "On-chip CMOS-compatible all-optical integrator”, Nature Communications, vol. 1, Article 29, 2010.

[203] A. Pasquazi, et al., “All-optical wavelength conversion in an integrated ring resonator,” Optics Express, vol. 18 (4) 3858 (2010).

[204] A. Pasquazi, Y. Park, J. Azana, et al., "Efficient wavelength conversion and net parametric gain via Four Wave Mixing in a high index doped silica waveguide," Optics Express, vol. 18, no. 8, pp. 7634-7641, 2010.

[205] M. Peccianti, M. Ferrera, L. Razzari, et al., "Subpicosecond optical pulse compression via an integrated nonlinear chirper," Optics Express, vol. 18, no. 8, pp. 7625-7633, 2010.

[206] Little, B. E. et al., "Very high-order microring resonator filters for WDM applications", IEEE Phot. Technol. Lett. 16, 2263(2004).

[207] M. Ferrera et al., "Low Power CW Parametric Mixing in a Low Dispersion High Index Doped Silica Glass MicroRing Resonator with Q-factor > 1 Million", Optics Express, vol.17, no. 16, pp. 14098-14103 (2009).

[208] M. Peccianti, et al., "Demonstration of an ultrafast nonlinear microcavity modelocked laser", Nature Comm., vol. $3,765,2012$.

[209] A. Pasquazi, et al., "Self-locked optical parametric oscillation in a CMOS compatible microring resonator: a route to robust optical frequency comb generation on a chip," Optics Express, vol. 21, no. 11, pp. 13333-13341, 2013.

[210] A. Pasquazi, et al., "Stable, dual mode, high repetition rate mode-locked laser based on a microring resonator," Optics Express, vol. 20, no. 24, pp. 27355-27362, 2012.

[211] Xu, X., et al., Photonic microwave true time delays for phased array antennas using a $49 \mathrm{GHz}$ FSR integrated microcomb source, Photonics Research, 6, B30-B36 (2018).

[212] X. Xu, M. Tan, J. Wu, R. Morandotti, A. Mitchell, and D. J. Moss, "Microcomb-based photonic RF signal processing”, IEEE Photonics Technology Letters, vol. 31 no. 23 1854-1857, 2019.

[213] M. Tan et al, "Orthogonally polarized Photonic Radio Frequency single sideband generation with integrated microring resonators", IOP Journal of Semiconductors, Vol. 42 (4), 041305 (2021). DOI: 10.1088/16744926/42/4/041305.

[214] Xu, et al., "Advanced adaptive photonic RF filters with 80 taps based on an integrated optical micro-comb source," Journal of Lightwave Technology, vol. 37, no. 4, pp. 1288-1295 (2019).

[215] X. Xu, et al., Broadband microwave frequency conversion based on an integrated optical micro-comb source", Journal of Lightwave Technology, vol. 38 no. 2, pp. 332-338, 2020.

[216] M. Tan, et al., "Photonic RF and microwave filters based on 49GHz and 200GHz Kerr microcombs", Optics Comm. vol. 465,125563, Feb. 22. 2020.

[217] X. Xu, et al., "Broadband photonic RF channelizer with 90 channels based on a soliton crystal microcomb", Journal of Lightwave Technology, Vol. 38, no. 18, pp. 5116 - 5121, 2020. doi: 10.1109/JLT.2020.2997699.

[218] X. Xu, et al., "Photonic RF and microwave integrator with soliton crystal microcombs", IEEE Transactions on Circuits and Systems II: Express Briefs, vol. 67, no. 12, pp. 3582-3586, 2020. DOI:10.1109/TCSII.2020.2995682.

[219] X. Xu, et al., "High performance RF filters via bandwidth scaling with Kerr micro-combs," APL Photonics, vol. 4 (2) 026102.2019.

[220] M. Tan, et al., "Microwave and RF photonic fractional Hilbert transformer based on a 50 GHz Kerr micro-comb", Journal of Lightwave Technology, vol. 37, no. 24, pp. 6097 - 6104, 2019.

[221] M. Tan, et al., "RF and microwave fractional differentiator based on photonics", IEEE Transactions on Circuits and Systems: Express Briefs, vol. 67, no.11, pp. 2767-2771, 2020. DOI:10.1109/TCSII.2020.2965158. 
[222] M. Tan, et al., "Photonic RF arbitrary waveform generator based on a soliton crystal micro-comb source", Journal of Lightwave Technology, vol. 38, no. 22, pp. 6221-6226 (2020). DOI: 10.1109/JLT.2020.3009655.

[223] M. Tan, X. Xu, J. Wu, R. Morandotti, A. Mitchell, and D. J. Moss, "RF and microwave high bandwidth signal processing based on Kerr Micro-combs", Advances in Physics X, VOL. 6, NO. 1, 1838946 (2021). DOI:10.1080/23746149.2020.1838946.

[224] X. Xu, et al., "Advanced RF and microwave functions based on an integrated optical frequency comb source," Opt. Express, vol. 26 (3) 2569 (2018).

[225] M. Tan, X. Xu, J. Wu, B. Corcoran, A. Boes, T. G. Nguyen, S. T. Chu, B. E. Little, R.Morandotti, A. Lowery, A. Mitchell, and D. J. Moss, ""Highly Versatile Broadband RF Photonic Fractional Hilbert Transformer Based on a Kerr Soliton Crystal Microcomb”, Journal of Lightwave Technology vol. 39 (24) 7581-7587 (2021).

[226] Bao, C., et al., Direct soliton generation in microresonators, Opt. Lett, 42, 2519 (2017).

[227] Yuning Zhang, Yang Qu, Jiayang Wu, Linnan Jia, Yunyi Yang, Xingyuan Xu, Baohua Jia, and David J. Moss, "Enhanced Kerr nonlinearity and nonlinear figure of merit in silicon nanowires integrated with 2D graphene oxide films", ACS Applied Materials and Interfaces, Vol. 12 (29) 33094-33103 (2020). DOI:10.1021/acsami.0c07852

[228] D.Moss, "11 Tera-FLOP/s photonic convolutional accelerator and deep learning optical neural networks", Research Square, (2021). DOI: https://doi.org/10.21203/rs.3.rs-493347/v1.

[229] Moss, David (2020): "11.0 Tera-FLOP/second photonic convolutional accelerator for deep learning optical neural networks", TechRxiv. Preprint. (2020). https://doi.org/10.36227/techrxiv.13238423.v1

[230] Xu, X.; Tan, M.; Corcoran, B.; Wu, J.; Boes, A.; Nguyen, T.; Chu, S.; Little, B.; Hicks, D.; Morandotti, R.; Mitchell, A.; Moss, D. “11 Tera-FLOP per Second Photonic Convolutional Accelerator for Deep Learning Optical Neural Networks", Preprints 2020, 2020110420.

[231] Moss, David (2020): "RF and microwave photonic high bandwidth signal processing based on Kerr micro-comb sources”, TechRxiv. (2020). Preprint. DOI:10.36227/techrxiv.12665609.v3

[232] Yuning Zhang, Jiayang Wu, Yunyi Yang, Yang Qu, Linnan Jia, Tania Moein, Baohua Jia, David J. Moss, "Enhanced nonlinear optical figure-of-merit at $1550 \mathrm{~nm}$ for silicon nanowires integrated with graphene oxide layered films", Arxiv (2020). arXiv:2004.08043 [physics.optics]

[233] Moss, David; Jia, Baohua; Wu, Jiayang; Zhang, Yuning; Yang, Yunyi; Jia, Linnan, Yang Qu, Tania Moein (2020): "Transforming silicon into a high performing integrated nonlinear photonics platform by integration with $2 \mathrm{D}$ graphene oxide films", TechRxiv. (2020). Preprint. DOI:10.36227/techrxiv.12061809.v1.

[234] A. Frigg, A. Boes, G. Ren, T.G. Nguyen, D.Y. Choi, S. Gees, D. Moss, A Mitchell, "Optical frequency comb generation with low temperature reactive sputtered silicon nitride waveguides", APL Photonics, Vol. 5 (1), 011302 (2020).

[235] M. Tan, X. Xu, J. Wu, A. Boes, B. Corcoran, T. G. Nguyen, S. T. Chu, B. E. Little, R. Morandotti, A. Mitchell, and D. J. Moss, “Advanced applications of Kerr mircocombs", Paper 11775-1. SPIE 11775, Integrated Optics: Design, Devices, Systems and Applications VI, (EOO21) OO107-8, Proc 1177504 (18 April 2021); Integrated Optics Conference, SPIE Optics and Optoelectronics Symposium, Prague, Czech Republic. April 19 - 22 (2021), doi.org/10.1117/12.2588733.

[236] T. Moein, D. Gailevičius, T. Katkus, S.H. Ng, S. Lundgaard, D.J. Moss, H. Kurt, Vygantas Mizeikis, Kęstutis Staliūnas, Mangirdas Malinauskas, Saulius Juodkazis, "Optically-thin broadband graphene-membrane photodetector", Nanomaterials, Vol. 10 (3), 407 (2020).

[237] Moss, David, "Microcombs for Ultrahigh Bandwidth Optical Data Transmission and Neural Networks." OSF Preprints. March 8. (2021). DOI:10.31219/osf.io/ne9wx.

[238] M. Tan, X. Xu, D. J. Moss, "Tera-OPs Photonic Convolutional Neural Networks based on Kerr Microcombs," Preprints, 2021. DOI: 10.20944/preprints202102.0549.v1.

[239] M. Tan, X. Xu, D.J. Moss, "Advanced applications of microcombs: From optical neural networks to data transmission," Research Square, April 2021. DOI: 10.21203/rs.3.rs-409803/v1.

[240] D. J. Moss et al.,'Tunable dispersion and dispersion slope compensators for $10 \mathrm{~Gb} / \mathrm{s}$ using all-pass multicavity etalons", IEEE Phot. Technology Letters, vol. 15, no. 5, 730-732 (2003).

[241] L.M. Lunardi et al. "Tunable dispersion compensators based on multi-cavity all-pass etalons for 40Gb/s systems", J. Lightwave Technology, vol. 20, (12) 2136 (2002).

[242] A. Della Torre et al., "Mid-infrared supercontinuum generation in a low-loss germanium-on-silicon waveguide", APL Photonics Vol. 6, 016102 (2021); doi: 10.1063/5.0033070.

[243] M. Sinobad, et al., "Mid-infrared supercontinuum generation in silicon-germanium all-normal dispersion waveguides”, Optics Letters, Vol. 45 (18), 5008-5011 (2020). DOI: 10.1364/OL.402159. 
[244] M. Sinobad et al., "High coherence at $\mathrm{f}$ and $2 \mathrm{f}$ of a mid-infrared supercontinuum in a silicon germanium waveguide", IEEE Journal of Selected Topics in Quantum Electronics Vol. 26 (2) 8201008 (2020). DOI:10.1109/JSTQE.2019.2943358.

[245] M. Sinobad et al., "Dispersion trimming for mid-infrared supercontinuum generation in a hybrid chalcogenide SiGe waveguide", Journal of the Optical Society of America B, Vol. 36 (2) A98-A104 (2019). DOI: 10.1364/JOSAB.36.000A98.

[246] M. Sinobad et al., "High brightness mid-infrared octave spanning supercontinuum generation to $8.5 \mu \mathrm{m}$ in chipbased Si-Ge waveguides", Optica, Vol. 5 (4) 360-366 (2018). DOI:10.1364/OPTICA.5.000360.

[247] L. Jin et al., Applied Physics Letters Photonics, Vol, 5 Article 056106, (2020). DOI:10.1063/5.0002941

[248] L. Carletti et al., "Nonlinear optical properties of Si-Ge waveguides in the mid-infrared", Optics Express Vol. 23 (7) 8261-8271 (2015). 